\title{
A Rules-Based Approach for Configuring Chains of Classifiers in Real-Time Stream Mining Systems
}

\author{
Brian Foo and Mihaela van der Schaar \\ Department of Electrical Engineering, University of California Los Angeles (UCLA), 66-147E Engineering IV Building, \\ 420 Westwood Plaza, Los Angeles, CA 90095, USA \\ Correspondence should be addressed to Brian Foo, brian.foo@gmail.com
}

Received 20 November 2008; Revised 8 April 2009; Accepted 9 June 2009

Recommended by Gloria Menegaz

\begin{abstract}
Networks of classifiers can offer improved accuracy and scalability over single classifiers by utilizing distributed processing resources and analytics. However, they also pose a unique combination of challenges. First, classifiers may be located across different sites that are willing to cooperate to provide services, but are unwilling to reveal proprietary information about their analytics, or are unable to exchange their analytics due to the high transmission overheads involved. Furthermore, processing of voluminous stream data across sites often requires load shedding approaches, which can lead to suboptimal classification performance. Finally, real stream mining systems often exhibit dynamic behavior and thus necessitate frequent reconfiguration of classifier elements to ensure acceptable end-to-end performance and delay under resource constraints. Under such informational constraints, resource constraints, and unpredictable dynamics, utilizing a single, fixed algorithm for reconfiguring classifiers can often lead to poor performance. In this paper, we propose a new optimization framework aimed at developing rules for choosing algorithms to reconfigure the classifier system under such conditions. We provide an adaptive, Markov model-based solution for learning the optimal rule when stream dynamics are initially unknown. Furthermore, we discuss how rules can be decomposed across multiple sites and propose a method for evolving new rules from a set of existing rules. Simulation results are presented for a speech classification system to highlight the advantages of using the rules-based framework to cope with stream dynamics.
\end{abstract}

Copyright (C) 2009 B. Foo and M. van der Schaar. This is an open access article distributed under the Creative Commons Attribution License, which permits unrestricted use, distribution, and reproduction in any medium, provided the original work is properly cited.

\section{Introduction}

A variety of real-time applications require complex topologies of operators to perform classification, filtering, aggregation, and correlation over high-volume, continuous data streams [1-7]. Due to the high computational burden of analyzing such streams, distributed stream mining systems have been recently developed. It has been shown that distributed stream mining systems transcend the scalability, reliability, and performance objectives of large-scale, realtime stream mining systems [5, 7-9]. In particular, many mining applications implement topologies of classifiers to jointly accomplish a complex classification task $[10,11]$. Such structures enable the application to leverage computational resources and analytics across different sites to provide dynamic filtering and successive identification of stream data.
Nevertheless, several key challenges remain for configuring networks of classifiers in distributed stream mining systems. First, real-time stream mining applications must cope effectively with system overload due to large data volumes, or limited system resources, while maintaining high classification performance (i.e., utility). A novel methodology was introduced recently for configuring the operating point (e.g., threshold) of each classifier based on its performance, as well as its output data rate, such that the joint configurations meet the resource constraints at all downstream classifiers in the topology while maximizing detection rate [11]. In general, such operation points exist for a majority of classification schemes, such as support vector machines, $k$-Nearest neighbors, Maximum Likelihood, and Random Decision Trees. While this methodology performs well when the relationships between classifier analytics are known (e.g., the exclusivity principle for filtering subset data 
Prior approaches
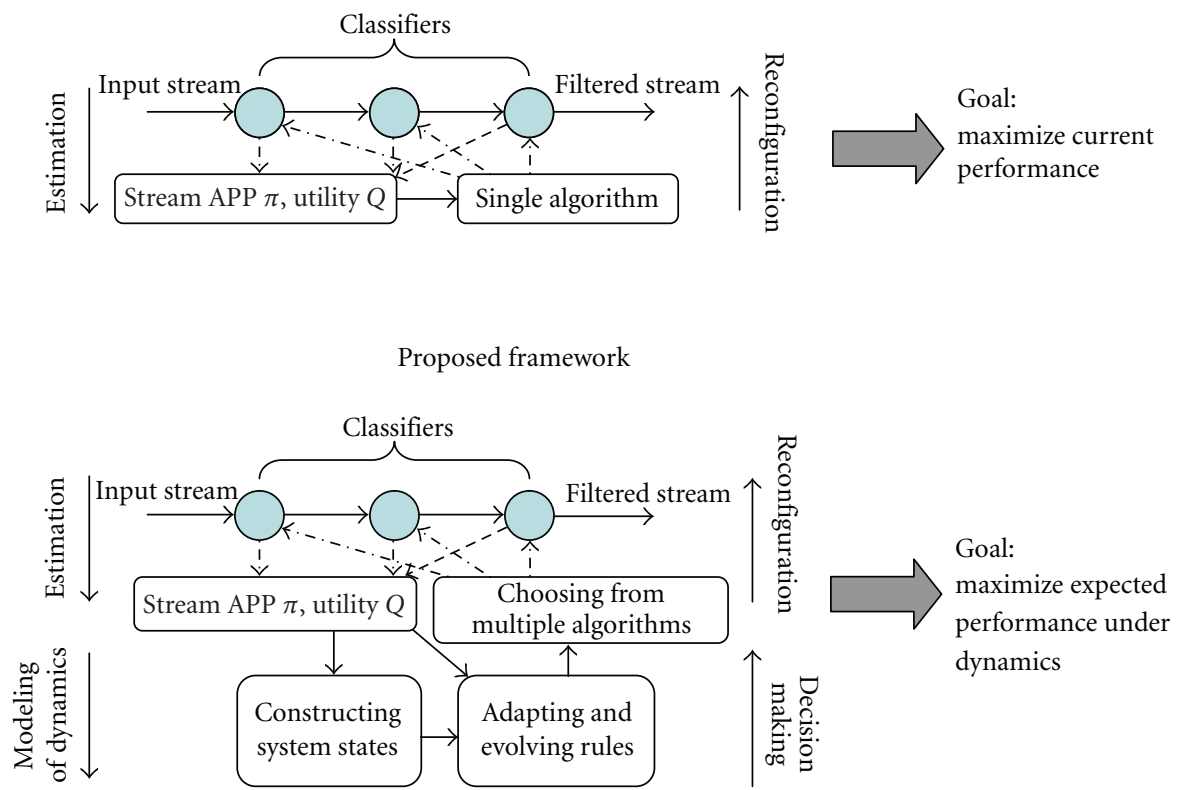

FiguRE 1: Comparison of prior approaches and the proposed rules-based framework.

from the previous classifier [11]), joint optimization between autonomous sites can be a very difficult problem, since the analytics used to perform successive classification/filtering may be physically distributed across sites owned by different companies $[7,12]$. These analytics may have complex relationships and often cannot be unified into a single repository due to legal, proprietary or technical restrictions [13, 14]. Second, data streams often have time-varying rates and characteristics, and thus, they require frequent reconfiguration to ensure acceptable classification performance. In particular, many existing algorithms optimally configure classifiers under fixed stream characteristics $[13,15]$. However, some algorithms can perform poorly when stream characteristics are highly time-varying. Hence, it becomes important to design rules or guidelines to determine for each classifier the best algorithm to use for reconfiguration at any given time, based on its short-term as well as long-term effects on future performance.

In this paper, we introduce a novel rules-based framework for configuring networks of classifiers in informationally distributed and dynamic environments. A rule acts as an instruction that determines for different stream characteristics, the proper algorithm to use, for classifier reconfiguration. We focus on a chain of binary classifiers as our main application [4], since chains of classifiers are easier to analyze, while offering flexibility in terms of configurations that can affect both the overall quality of classification as well as the end-to-end processing delay. Figure 1 depicts the proposed framework compared to prior approaches for reconfiguring chains of classifiers. The main features are highlighted as follows. (i) Estimation. Important local information, such as the estimated a priori probabilities (APP) of positive data from the input stream at each classifier and processing resource constraints, is gathered to determine the utility of the stream processing system. In our prior work, we introduced a method for distributed information gathering, where each classifier summarizes its local observations using several scalar values [13]. The values can be exchanged between nodes in order to obtain an accurate estimate of the overall stream processing utility, while keeping the communications overhead low and maintaining a high level of information privacy across sites.

(ii) Reconfiguration. Classifier reconfiguration can be performed by using an algorithm that analytically maximizes the stream processing utility based on the processing rate, accuracy, and delay. Note that while, in some cases, a centralized scheme can be used to determine the optimal configuration [11], in informationally distributed environments, it is often impossible to determine the performance of an algorithm until sufficient time is given to estimate the accuracy/delay of the processed data [13]. Such environments require the use of randomized or iterative algorithms that converge to the optimal configuration over time. However, when the stream is dynamic, it often does not make sense to use an algorithm that configures for the current time interval, since stream characteristics may have changed during the next time interval. Hence, having multiple algorithms available enables us to choose the optimal algorithm based on the expected stream behavior in future time intervals.

(iii) Modeling of Dynamics. To determine the optimal algorithm for reconfiguration, it is necessary to have a model of 
stream dynamics. Stream dynamics affect the APP of positive data arriving at each classifier, which in turn affects each classifier's local utility function. In our work, we define a system state to be a quantized value over each classifier's local utility values as well as the overall stream processing utility. We propose a Markov-based approach to model state transitions over time as a function of the previous state visited and algorithm used. This model enables us to choose the algorithm that leads to the best expected system performance in each system state.

(iv) Rules-Based Decision-Making. We introduce the concept of rules, where a rule determines the proper algorithm to apply for system reconfiguration in each state. We provide an adaptive solution for using rules when stream characteristics are initially unknown. Each rule is played with a different probability, and the probability distribution is adapted to ensure probabilistic convergence to an optimal steady state rule. Furthermore, we provide an efficiency bound on the performance of the convergent rule when a limited number of iterations are used to estimate stream dynamics (i.e., imperfect estimation). As an extension, we also provide an evolutionary approach, where a new rule is generated from a set of old rules based on the best expected utility in the following time interval based on modeled dynamics. Finally, we discuss conditions under which a large set of rules can be decomposed into small sets of local rules across individual classifier sites, which can then make autonomous decisions about their locally utilized algorithms.

While dynamic, resource-constrained, and distributed classification is an application that very well highlights the merits of our approach, we note that the framework developed in this paper can also be applied to any application that meets the following two criteria: (a) the utility can be measured and estimated by the system during any given time interval, but (b) the system cannot directly reconfigure and reoptimize due to unknown dynamics in system resource availabilities and application data characteristics. Importantly, in contrast to existing works that develop solutions for specific application domains such as optimizing classifier trees [16] or resource-constrained/delay-sensitive data processing [17], we are proposing a method that encapsulates such existing algorithms and determines rules on when to best apply them based on system and application dynamics.

This paper is organized as follows. In Section 2, we review several related works that address various challenges in distributed, resource-constrained stream mining systems, and decision-making in dynamic environments. In Section 3, we introduce the application of interest, which is optimizing distributed classifier chains, and propose a delay-sensitive utility function. We also discuss a distributed information gathering approach to estimate the utility when each site is unwilling to share proprietary data. In Section 4, we introduce the rules-based framework for choosing algorithms to apply under different system conditions. Extensions to the rules-based framework, such as the decomposition of rules across distributed classifier sites and evolving a new rule from existing rules, are discussed in Section 5. Simulation results from a speech classification application are given in Section 6, and conclusions in Section 7.

\section{Review of Existing Works}

2.1. Resource-Constrained Classification. Various works in resource-constrained stream mining deal with both valueindependent and value-dependent load shedding schemes. Value independent (or probabilistic) load shedding solutions [17-22] perform well for simple data management jobs such as aggregation, for which the quality depends only on the sample size. However, this approach is suboptimal for applications where the quality is value-dependent, such as the confidence level of data in classification. A valuedependent load shedding approach is given in $[11,15]$ for chains of binary filtering classifiers, where each classifier configures its operating point (e.g., threshold) based on the quality of classification as well as the resource availability across utilized processing nodes. However, in order to analytically optimize the quality of joint classification, strong assumptions about the relations between classifiers are often required (e.g., exclusivity [11], where each chained classifier filters out a subset of data from the previous classifier). Such assumptions about classifier relationships may not be valid when each classifier is independently trained and placed on different sites owned by different companies.

A recent work that considers stream dynamics involves intelligent load shedding for a classifier [23], where the load shedder attempts to maximize certain Quality of Decision (QoD) measures based on the predicted distribution of feature values in future time units. However, this work focuses mainly on load shedding for a single classifier rather than a distributed network of classifiers. Without a joint consideration of resource constraints and effects on feature values at downstream classifiers, the quality of classification can suffer, and the end-to-end processing delay can become intolerable for real-time applications $[24,25]$.

Finally, in our prior work [13], we proposed a modelfree experimentation solution to maximize the performance of a delay-sensitive stream mining application using a chain of resource-constrained classifiers. (We provide a brief tutorial on delay-sensitive stream mining with a chain of classifiers in Section 3.) We proved that this solution converged to the optimal configuration for static streams, even when the relationships between individual classifier analytics are unknown. However, the experimentation solution could not provide any performance guarantees for dynamic streams. Importantly, in the above works, dynamics and information-decentralization have been addressed in isolation for resource-constrained classification, but there has not been an integrated framework to address these challenges jointly.

\subsection{Markov Decision Process versus Rules-Based Decision} Making. In addition to distributed stream mining, related works exist for decision-making in dynamic environments. A widely used framework for optimizing the performance 
of dynamic systems is the Markov decision process (MDP) [26], where a Markov model is used for state transitions as a function of the previous state and action (e.g., configuration) taken. In an MDP framework, there exists an optimal policy (i.e., a function mapping states to actions) that maximizes an expected value function, which is often given as the sum of discounted future rewards (e.g., expected utilities at future time intervals). When state transition probabilities are unknown, reinforcement learning techniques can be applied to determine the optimal policy, which involves a delicate balance between exploitation (playing the action that gives the highest estimated value) and exploration (playing an action of suboptimal value) [27].

While our rules-based framework is derived from the MDP framework (e.g., rules map states to algorithms while policies map states to actions), there is a key difference between traditional MDP-based approaches and our proposed rules-based approach. Unlike the MDP framework, where actions must be specified by quantized (discrete) configurations, algorithms are explicitly designed to perform iterative optimization over previous configurations [28]. Hence, their outputs are not limited to a discrete set of configurations/actions, but rather converge to a locally or globally optimal configuration over the real (continuous) space of configurations. Furthermore, algorithms avoid the complication involving how the configurations (actions) should be quantized in dynamic environments, for example, when stream characteristics change over time.

Finally, there have been recent advances in collaborative multiagent learning between distributed sites related to our proposed work. For instance, the idea of using a playbook to select different rules or strategies and reinforcing these rules/strategies with different weights based on their performances, is proposed in [29]. However, while the playbook proposed in [29] is problem specific, we envision a broader set of rules capable of selecting optimization algorithms with inherent analytical properties leading to utility maximization of not only stream processing but also distributed systems in general. Furthermore, our aim is to construct a purely automated framework for both information gathering and distributed decision making, without requiring supervision, as supervision may not be possible across autonomous sites or can lead to high operational costs.

\section{Background on Binary Classifier Chains}

3.1. Characterizing Binary Classifiers and Classifier Chains. A binary classifier partitions input data objects into two classes, a "yes" class $\mathrm{H}$ and a "no" class $\overline{\mathrm{H}}$. A binary classifier chain is a special case of a binary classifier tree, where multiple binary classifiers are used to detect the intersection of multiple classes of interest. In particular, the outputs stream data objects (SDOs); the "yes" class of a classifier, are fed as inputs to the successive classifier in the chain [11], such that the entire chain acts as a serial concatenation of data filters. For simplicity of notation, we index each binary classifier in the chain by $v_{i}, i=1, \ldots, I$, in the order that it processes an input stream, as shown in Figure 2. Data objects that are classified as "no" are dropped from the stream.

Given the ground truth $X_{i}$ for an input SDO to classifier $v_{i}$, denote the classification decision on the SDO by $\hat{X}_{i}$. The proportion of correctly forwarded samples is captured by the probability of detection $P_{i}^{D}=\operatorname{Pr}\left\{\hat{X}_{i} \in \mathrm{H}_{i} \mid X_{i} \in \mathrm{H}_{i}\right\}$, and the proportion of incorrectly forwarded samples is captured by the probability of false alarm $P_{i}^{F}=\operatorname{Pr}\left\{\hat{X}_{i} \in\right.$ $\left.\mathrm{H}_{i} \mid X_{i} \notin \mathrm{H}_{i}\right\}$. Each classifier $v_{i}$ can be characterized by a detection-error-tradeoff (DET) curve or a curve that maps the false alarm configuration $P_{i}^{F}$ to a probability of detection $P_{i}^{D}[30,31]$. For instance, a DET curve can be mapped out by different thresholds on the output scores of a support vector machine [32]. A typical DET curve is shown in Figure 3. Due to the functional mapping from false alarm to detection probabilities and also to maintain a representation that can be generalized over many types of classifiers, we denote the configuration of each classifier by its false alarm probability $P_{i}^{F}$. The vector of false alarm configurations for the entire chain is denoted $\mathbf{P}^{F}$.

3.2. A Utility Function for a Chain of Classifiers. The goal of a stream processing application is to maximize not only the amount of processed data (the throughput), but also the amount of data that is correctly processed by each classifier (the goodput). However, increasing the throughput also leads to an increased load on the system, which increases the end-to-end delay for the stream. We can determine the performance and delay based on the following metrics. Suppose that the input stream to classifier $v_{i}$ has a priori probability (APP) $\pi_{i}$ of being in the positive class. The probability of labeling an SDO as positive can be given by

$$
\ell_{i}=\pi_{i} P_{i}^{D}+\left(1-\pi_{i}\right) P_{i}^{F} .
$$

The probability of correctly labeling an SDO as positive can be given by

$$
\wp_{i}=\pi_{i} P_{i}^{D}
$$

For a chain of classifiers as shown in Figure 2, the end-to-end cost can be given by

$$
\begin{aligned}
C & =(\pi-\wp)+\theta(\ell-\wp) \\
& =\pi-\prod_{i=1}^{n} \wp_{i}+\theta\left(\prod_{i=1}^{n} \ell_{i}-\prod_{i=1}^{n} \wp_{i}\right),
\end{aligned}
$$

where $\pi$ indicates the true APP of input data that belongs to the intersection of all positive classes of the classifiers, and $\theta$ specifies the cost of false positives relative to true positives. Since $\pi$ depends only on the stream characteristics, we can regard it as constant and remove it from the cost function, invert it, and produce a utility function: $F=\prod_{i=1}^{n} \wp_{i}-$ $\theta\left(\prod_{i=1}^{n} \ell_{i}-\prod_{i=1}^{n} \wp_{i}\right)[13,15]$. Note that $\prod_{i=1}^{n} \ell_{i}$ is simply the total fraction of stream data forwarded across the entire chain. $\prod_{i=1}^{n} \wp_{i}=\prod_{i=1}^{n} \pi_{i} P_{i}^{D}$, on the other hand, is the fraction of data out of the entire stream that is correctly forwarded across the entire chain, which is calculated by the probability 
TABLE 1: Summary of parameter types and a few examples.

\begin{tabular}{llc}
\hline Type of parameter for $v_{i}$ & Description & Examples \\
\hline Static parameters & Fixed parameters, exchanged during initialization & $\pi$ \\
Observed parameters & Can be measured by the last classifier $v_{n}$ & $D$ \\
Exchanged parameters & Traded with other classifiers & $\wp_{i}, \ell_{i}$ \\
Configurable parameters & Configured by classifier $v_{i}$ & $P_{i}^{F}$ \\
\hline
\end{tabular}

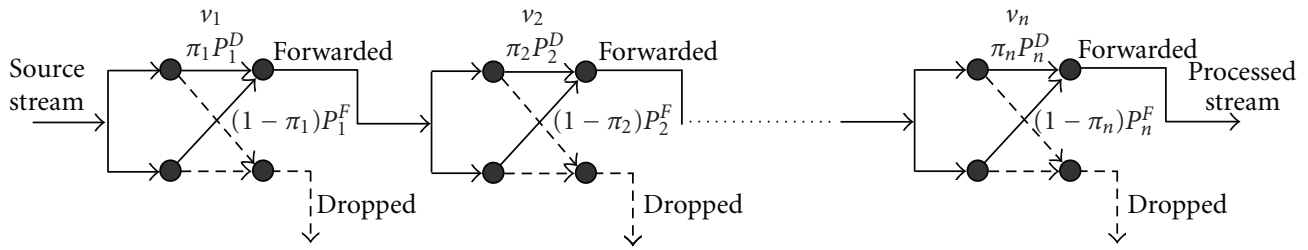

Figure 2: Classifier chain with probabilities labeled on each edge.

of detection by each classifier, times the conditional APP of positive data at the input of each classifier $v_{i}$.

To factor in the delay, we consider an end-to-end processing delay penalty $G(D)=e^{-\varphi D}$, where $\varphi$ reflects the application's delay sensitivity $[24,25]$, with large $\varphi$ indicating that the application is highly delay sensitive, and small $\varphi$ indicating that the delay on processed data is unimportant. Note that this function not only has an important meaning as a discount factor in game theoretic literature [26] but also can also be analytically derived by modeling each classifier as an $M / M / 1$ queuing facility often used for networks and distributed stream processing systems [33, 34]. Denote the total SDO input rate and the processing rate for each classifier $v_{i}$, by $\lambda_{i}$ and $\mu_{i}$, respectively. Note furthermore from (1) that each classifier acts as a filter that drops each SDO with i.i.d. probability $1-\ell_{i}$, and forwards the SDO with i.i.d. probability $\ell_{i}$ to the next-hop classifier, based on its operating point on the DET curve. The resulting output to each nexthop classifier is also given by a Poisson process [35], where the arrival rate of input data to classifier $v_{i}$ is given by $\lambda_{i}=\lambda_{0} \prod_{j=1}^{i-1} \ell_{j}$. Because the output of an $M / M / 1$ system has i.i.d. interarrival times, the delays for each classifier in a classifier system, given the arrival and service rates, are also independent [36]. Hence, the expected delay penalty $G(D)$ for the entire chain can be calculated from the moment generating function [37]:

$$
E[G(D)]=\Phi_{D}(-\varphi)=\prod_{i=1}^{n}\left(\frac{\mu_{i}-\lambda_{i}}{\mu_{i}-\lambda_{i}+\varphi}\right) .
$$

In order to combine the two different objectives (accuracy and delay), we construct a single objective function $F$. $G(D)$, based on the concept of fairness implemented by the Nash product [38]. (The generalized Nash product provides a tradeoff between misclassification cost $[15,39]$ and delay depending on the exponent attached to each term $F^{\alpha}$ and $H(D)^{(1-\alpha)}$, respectively. In practice, we observed through simulations that, for the considered applications, an equal weight $\alpha=0.5$ provided the best tradeoff between classification accuracy and delay.) The overall utility of realtime stream processing is therefore

$$
\begin{aligned}
& \max _{\mathbf{P}^{F} \forall v_{i} \in V} Q\left(\mathbf{P}^{F}\right)=\max _{\mathbf{P}^{F}} G(D)\left(\prod_{i=1}^{n} \wp_{i}-\theta\left(\prod_{i=1}^{n} \ell_{i}-\prod_{i=1}^{n} \wp_{i}\right)\right) \\
& \text { s.t. } \quad \mathbf{0} \leq \mathbf{P}^{F} \leq \mathbf{1} .
\end{aligned}
$$

3.3. Information-Distributed Estimation of Stream Processing Utility. Note that while classifiers may be willing to provide information about $P_{i}^{F}$ and $P_{i}^{D}$, the conditional APP $\pi_{i}$ at every classifier $v_{i}$ is, in general, a complicated function of the false alarm probabilities of all previous classifiers, that is, $\pi_{i}=\pi_{i}\left(\mathbf{P}_{j}^{F}\right)_{j<i}$. This is because setting different thresholds for the false alarm probabilities at previous classifiers will affect the incoming source distribution to classifier $v_{i}$. One way to visualize this effect is to consider a Gaussian mixture model operated on by a chain of 2 linear classifiers, where changing the threshold of the first classifier will affect the positive and negative data distribution of the second classifier. However, because analytics trained across different sites may not obey simple relationships (e.g., subsets), constructing a joint classification model is very difficult if sites do not share their analytics. Due to legal and proprietary restrictions, it can be assumed that, in practice, the joint model cannot be constructed, and hence the objective function $Q\left(\mathbf{P}^{F}\right)$ is unknown.

While the precise form of $Q\left(\mathbf{P}^{F}\right)$ is unknown and is most likely changing due to stream dynamics, the utility can still be estimated over a short time interval if classifier configurations are held fixed over the length of the interval. This is discussed in more detail in our prior work and summarized in Figure 4 . First, the average service rate $\mu_{i}$ is fixed (static) for each classifier and can be exchanged with other classifiers upon system initialization. Second, the arrival rate into classifier $v_{i}, \lambda_{i}$, can be obtained by simply measuring (or observing) the number of SDOs in the input stream. Finally, the goodput and throughput ratios $\wp_{i}$ and $\ell_{i}$ are functions of the configuration $P_{i}^{F}$ and the 


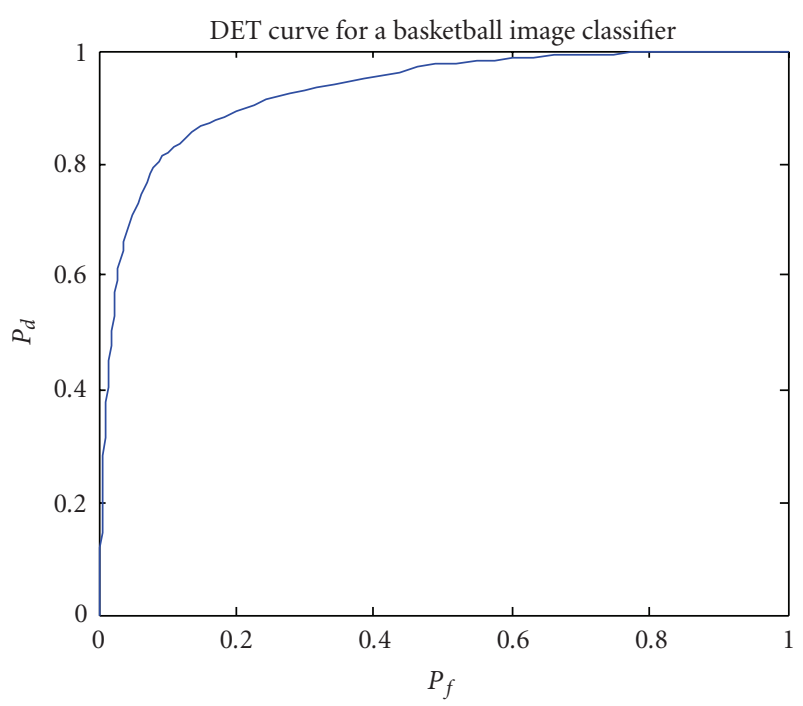

Figure 3: The DET curve for an image classifier used to detect basketball images [40].

APP. The APP can be estimated from the input stream using maximum a priori (MAP) schemes. Consequently, every parameter in (5) can be easily estimated based on some locally observable data. By exchanging these locally obtained parameters and configurations across all classifiers, each classifier can then estimate the overall stream processing utility. Table 1 summarizes the various parameter types, their descriptions, and examples in our problem.

\section{A Rules-Based Framework for Choosing Algorithms}

4.1. States, Algorithms, and Rules. Now that we have discussed the estimation portion of our framework (Figure 1), we move to discuss the proposed decision-making process in dynamic environments. We introduce the rules-based framework for choosing algorithms as follows.

(i) A set of states $\&=\left\{S_{1}, \ldots, S_{M}\right\}$ that capture information about the environment (e.g., APPs of input streams to each classifier) or the stream processing utility (local or global) and can be represented by quantized bins over these parameters.

(ii) The expected utility derived in each state $S_{m}, Q\left(S_{m}\right)$.

(iii) A set of algorithms $\mathcal{A}=\left\{A_{1}, \ldots, A_{K}\right\}$ that can be used to reconfigure the system, where an algorithm determines the configuration at time $t, \mathbf{P}_{t}^{F}$, based on prior configurations, for example, $\mathbf{P}_{t}^{F}=A_{k}\left(\mathbf{P}_{t-1}^{F}, \ldots, \mathbf{P}_{t-\tau}^{F}\right)$. Note that an algorithm differs from an action in the MDP framework [26] in that an action simply corresponds to a (discrete) fixed configuration. In fact, algorithms are generalizations of actions, since an action can be interpreted as an algorithm that always returns the same configuration regardless of the prior configurations, that is, $A_{k}\left(\mathbf{P}_{t-1}^{F}, \ldots, \mathbf{P}_{t-\tau}^{F}\right)=$ $\mathbf{c}_{k}$, where $\mathbf{c}_{k}$ is some constant configuration. (iv) A set of pure rules $\mathcal{R}=\left\{R_{1}, \ldots, R_{H}\right\}$. Each rule $R_{h}: \& \rightarrow \mathcal{A}$ is a deterministic mapping from a state to an algorithm, where the expression $R_{h}(S)=$ $A \in \mathcal{A}$ indicates that algorithm $A$ should be used if the current system state is $S$. Additionally, we introduce the concept of a mixed ruleR, which is a random rule with a probability distribution over the set of pure rules $\mathcal{R}$, given by a probability vector $\mathbf{r}=\left[p\left(R_{1}\right), \ldots, p\left(R_{H}\right)\right]^{T}$. For convenience, we denote a mixed rule by the dot product between the probability vector and the (ordered) set of pure rules, $\mathbf{r} \cdot \mathcal{R}=\sum_{h=1}^{H} \mathbf{r}_{h} R_{h}$, where $\mathbf{r}_{h}$ is the $h$ th element of $\mathbf{r}$. As will be shown later, mixed rules are powerful for both proving convergence results and for designing solutions to find the optimal rule for algorithm selection when stream characteristics are initially unknown.

4.2. State Spaces and Markov Modeling for Algorithms. Markov processes have been used extensively to model the behavior of dynamic streams (such as multimedia) due to their ability to capture temporal correlations of varying orders [23, 41]. In this section, we extend Markov modeling to the space of algorithms and rules. (Though a Markov model may not be entirely accurate for relating stream dynamics to algorithms, we provide evidence in our simulations that, for temporally-correlated stream data, the Markov model approximates the real process closely.) Importantly, based on Markov assumptions about algorithms and states, we can apply results from the MDP framework to show that the optimal rule for selecting algorithms in steady state is always pure. While this result is a simple consequence of the MDP framework, we provide a short proof below to guide us (in the following section) on how to construct a solution for learning the optimal pure rule under unknown stream dynamics. Moreover, the details in the proof will also enable us to prove efficiency bounds when stream parameters cannot be perfectly estimated.

Definition 1. Define a first-order algorithmic Markov process (or algorithmic Markov system) for a set of algorithms $\mathcal{A}$ and discrete state space quantization $\&$ as follows: the state and algorithm used at time $t,\left(s_{t}, a_{t}\right) \in \delta \times \mathcal{A}$, is a sufficient statistic for $s_{t+1}$. Hence, $s_{t+1}$ can be described by a probability transition function $p\left(s_{t+1} \mid s_{t}, a_{t}\right)=$ $p\left(s_{t+1} \mid s_{t}, a_{t}\left(\mathbf{P}_{t-1}^{F}, \ldots, \mathbf{P}_{t-\tau}^{F}\right)\right)$ for any past configurations $\left(\mathbf{P}_{t-1}^{F}, \ldots, \mathbf{P}_{t-\tau}^{F}\right)$.

Note that Definition 1 implies that in the algorithmic Markov system model, the state transitions are not dependent on the precise configurations used in previous time intervals, but only on the algorithm and state visited during the last time interval.

Definition 2. Thetransition matrix for a pure rule $R_{h}$ over the set of states $\delta$ is defined as a matrix $\mathbf{P}\left(R_{h}\right)$ with entries $\left[\mathbf{P}\left(R_{h}\right)\right]_{i j}=p\left(s_{t+1}=S_{i} \mid s_{t}=S_{j}, a_{t}=R\left(s_{t}\right)\right)$. The transition matrix for a mixed rule $\mathbf{r} \cdot \mathcal{R}$ is given by a matrix 


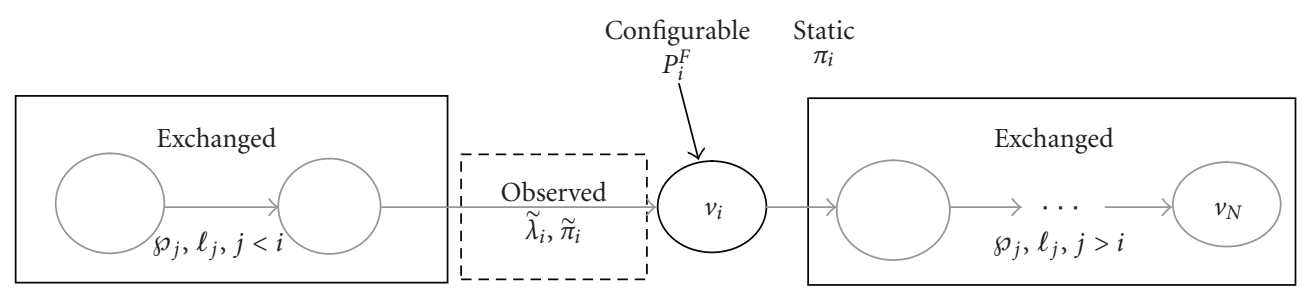

FIGURE 4: The various parameters in relation to $v_{i}$.

$\mathbf{P}(\mathbf{r} \cdot \mathcal{R})$ with entries: $[\mathbf{P}(\mathbf{r} \cdot \mathcal{R})]_{i j}=\sum_{h=1}^{H} \mathbf{r}_{h} p\left(s_{t+1}=S_{i} \mid\right.$ $\left.s_{t}=S_{j}, a_{t}=R_{h}\left(s_{t}\right)\right)$, where the subscript $h$ indicates the $h$ th component of $\mathbf{r}$. Consequently, the transition matrix for a mixed rule can also be written as $\mathbf{P}(\mathbf{r} \cdot \mathcal{R})=$ $\sum_{h=1}^{\mathscr{H}} \mathbf{r}_{h} \mathbf{P}\left(R_{h}\right)$.

Definition 3. The steady state distribution for being in each state $S_{m}$, given a rule $R_{h}$, is given by $p\left(s_{\infty}=S_{m}\right.$ । $\left.R_{h}\right)=\lim _{t \rightarrow \infty}\left[\mathbf{P}^{t}\left(R_{h}\right) \cdot \mathbf{e}\right]_{m}$, where $\mathbf{e}=[1,0, \ldots, 0]^{T}$. (Note that the steady state distribution can be efficiently calculated by finding the eigenvector corresponding to the largest eigenvalue (e.g., 1) of transition matrix $\mathbf{P}\left(R_{h}\right)$.) This can be conveniently expressed as a steady state distribution vector $\mathbf{p}_{\mathrm{ss}}\left(R_{h}\right)=\lim _{t \rightarrow \infty} \mathbf{P}^{t}\left(R_{h}\right) \cdot \mathbf{e}$.

Likewise, denote the utility vector for each state by $\mathbf{q}(\&)=\left[Q\left(S_{1}\right), \ldots, Q\left(S_{M}\right)\right]^{T}$. The steady-state average utility is given by

$$
Q\left(\mathbf{p}_{\mathrm{ss}}\left(R_{h}\right) \cdot \delta\right) \triangleq \mathbf{p}_{\mathrm{ss}}\left(R_{h}\right)^{T} \mathbf{q}(\&)
$$

Lemma 1. The steady state distribution for a mixed rule can be given as a linear function of the steady state distribution of pure rules, $\mathbf{p}_{s s}(r \cdot \mathcal{R})=\sum_{h=1}^{H} \mathbf{r}_{h} \mathbf{p}_{s s}\left(R_{h}\right)$. Likewise, the steady state average utility for a mixed rule can be given by $Q\left(\mathbf{p}_{s s}(\mathbf{r} \cdot \mathcal{R}) \cdot \delta\right)=\sum_{h=1}^{H} \mathbf{r}_{h} \mathbf{p}_{s s}\left(R_{h}\right)^{T} \mathbf{q}(\&)$.

Proof. The steady state distribution vector for being in each state can be derived by the following sequence of equations:

$$
\begin{aligned}
\mathbf{p}_{s s}(r \cdot \mathcal{R}) & =\lim _{t \rightarrow \infty} \mathbf{P}^{t}(\mathbf{r} \cdot \mathcal{R}) \cdot \mathbf{e} \\
& =\lim _{t \rightarrow \infty} \sum_{h=1}^{H} \mathbf{r}_{h} \mathbf{P}^{t}\left(R_{h}\right) \cdot \mathbf{e} \\
& =\sum_{h=1}^{H} \mathbf{r}_{h} \lim _{t \rightarrow \infty}\left[\mathbf{P}^{t}\left(R_{h}\right) \cdot \mathbf{e}\right] \\
& =\sum_{h=1}^{H} \mathbf{r}_{h} \mathbf{p}_{s \mathrm{ss}}\left(\mathrm{R}_{h}\right) .
\end{aligned}
$$

Likewise, the steady state average utility for a mixed rule can be given by

$$
\begin{aligned}
Q\left(\mathbf{p}_{\mathrm{ss}}(\mathbf{r} \cdot \mathcal{R}) \cdot \mathcal{8}\right) & =\sum_{m=1}^{M}\left[\sum_{h=1}^{H} \mathbf{r}_{h} p_{\mathrm{ss}}\left(s \mid R_{h}\right)\right] Q\left(S_{m}\right) \\
& =\sum_{h=1}^{H} \mathbf{r}_{h} \sum_{m=1}^{M} p_{\mathrm{ss}}\left(S_{m} \mid R_{h}\right) Q\left(S_{m}\right) \\
& =\sum_{h=1}^{H} \mathbf{r}_{h} \mathbf{p}_{\mathrm{ss}}\left(R_{h}\right)^{T} \mathbf{q}(\&) .
\end{aligned}
$$

Proposition 1. Given an algorithmic Markov system, a set of pure rules $\mathcal{R}$ and the option to play any mixed rule $\mathbf{r} \cdot \mathcal{R}$, the optimal rule in steady state is always pure. (Note that this proposition is proven in [26] for MDPs.)

Proof. The optimal mixed rule $\mathbf{r} \cdot \mathcal{R}$ in steady state maximizes the expected utility, which is obtained by solving the following problem:

$$
\begin{aligned}
& \max _{\mathbf{r}} Q\left(\mathbf{p}_{\mathrm{ss}}(\mathbf{r} \cdot \mathcal{R}) \cdot \wp\right) \\
& \text { s.t. } \quad \sum_{h=1}^{H} \mathbf{r}_{h}=1, \quad \mathbf{r} \geq \mathbf{0} .
\end{aligned}
$$

From Lemma 1, $Q\left(\mathbf{p}_{\mathrm{ss}}(\mathbf{r} \cdot \mathcal{R}) \cdot \&\right)=\sum_{h=1}^{H} \mathbf{r}_{h} \mathbf{p}_{\mathrm{ss}}\left(R_{h}\right)^{T} \mathbf{q}(\&)$, which is a linear transformation on the pure rule steady state distributions. Hence, the problem in (9) can be reduced to the following linear programming problem:

$$
\begin{gathered}
\max _{\mathbf{r}} \sum_{h=1}^{H} \mathbf{r}_{h} \mathbf{p}_{\mathrm{ss}}\left(R_{h}\right)^{T} \mathbf{q}(\&) \\
\sum_{h=1}^{H} \mathbf{r}_{h}=1, \quad \mathbf{r} \geq \mathbf{0} .
\end{gathered}
$$

Note that the extrema of the feasible set are given by points where only one component of $\mathbf{r}$ is 1 , and all other components are 0 , which correspond to pure rules. Since an optimal linear programming solution always exists at an extremum, there always exists an optimal pure rule in steady state. 
4.3. An Adaptive Solution for Finding the Optimal Pure Rule. We have shown in the previous section that an optimal rule is always pure under the Markov assumption. However, a mixed rule is often useful for estimating stream dynamics when the distribution of stream data values is initially unknown. For example, when a new application is run on a distributed stream mining system, there may not be any prior transmitted information about its stream statistics (e.g., average data rate, APPs for each classifier). In this section, we propose a solution called Simultaneous Parameter Estimation and Rule Optimization (SPERO). SPERO attempts to accomplish two important objectives. First, SPERO accurately estimates the state utilities and state transition probabilities, such that it can determine the optimal steady state pure rule from (10). Secondly, SPERO utilizes a mixed rule that not only approaches the optimal rule in the limit but also provides high performance during any finite time interval.

The description of the SPERO algorithm is as follows (highlighted in Figure 5). First each rule is initialized to be played with equal probability (this is the initial state of the top right box in Figure 5). After a rule is selected, the rule is used to choose an algorithm in the current system state, and the algorithm is applied to reconfigure the system. The result can be measured during the next time interval, and the system can then determine its next state as well as the resulting state utility. This information is updated in the Markov state space modeling box in Figure 5. After the state transition probabilities and state utilities are updated, expected utility in steady state is updated for each rule, and the optimal rule is chosen andreinforced. Reinforcement is simply increasing the probability of playing a rule that is expected to lead to the highest steady state utility, given the current estimation of state utilities and transition probabilities.

Algorithm 1 uses a slow reinforcement rate (increasing the probability that the optimal rule is played by the $m$ th root of the number of times it has been chosen as optimal), in order to guarantee steady state convergence to the optimal rule (Proof is given in the appendix). For visualization, in Figure 6 we plotted the mixed rules distribution chosen by SPERO for a set of 8 rules used in our simulations (see Section 6, Approach B for more details).

4.4. Tradeoff between Accuracy and Convergence Rate. In this section, we discuss the tradeoff between the estimation accuracy and the convergence rate of SPERO. In particular, SPERO uses a slow reinforcement rate to guarantee perfect estimation of parameters as $t \rightarrow \infty$. In practice however, it is often important to discover a good rule within a finite number of iterations, without continuing to sample rules that lead to states with poor performances. However, choosing a rule under finite observations can prevent the system from obtaining a perfect estimation of state utilities and transition probabilities, thereby converging to a suboptimal pure rule. In this section, we provide a probabilistic bound on the inefficiency of the convergent pure rule with respect to imperfect estimation caused by limited observations of each system state.
Consider when the real expected utility in a state is given by $Q\left(S_{m}\right)$, and the estimation based on time averaging of observations is given by $\hat{Q}\left(S_{m}\right)$. Depending on the variance of utility observations in that state $\sigma_{m}^{2}$, we can provide a probabilistic bound on achieving an estimation error of $\sigma$ with probability at least $1-\sigma_{m}^{2} / \sigma^{2}$ using Chebyshev's inequality, that is, $\operatorname{Pr}\left\{\left|Q\left(S_{m}\right)-\hat{Q}\left(S_{m}\right)\right| \geq \sigma\right\} \leq \sigma_{m}^{2} / \sigma^{2}$. Likewise, a similar probability estimation bound exists for the state transition probabilities, that is, $\operatorname{Pr}\left\{\mid \mathbf{P}_{i j}\left(R_{h}\right)-\right.$ $\left.\widetilde{\mathbf{P}}_{i j}\left(R_{h}\right) \mid \geq \delta\right\} \leq \eta$. Both of these bounds enable us to estimate the number of visits required in each state to discover an efficient rule within high probability. We provide the following proposition and corollary to determine an upper bound on the expected number of iterations required by SPERO to discover a near optimal rule.

Proposition 2. Suppose that $\left|Q\left(S_{m}\right)-\hat{Q}\left(S_{m}\right)\right| \leq \sigma$ and $\left|\mathbf{P}_{i j}\left(R_{h}\right)-\widetilde{\mathbf{P}}_{i j}\left(R_{h}\right)\right| \leq \delta$. Then the steady state utility of the convergent rule deviates from the utility of the optimal rule by no more than approximately $2 M \delta\left(U_{Q}+2 M \sigma\right)$, where $U_{Q}$ is the average system utility of the highest utility state.

Proof. From [42], it is shown that if the entry wise error of the probability transition matrices is $\delta$, then the steady state probabilities for the estimated and real transition probabilities obey the following relation:

$$
\begin{aligned}
\frac{\left|p_{\mathrm{ss}}\left(S_{m} \mid R_{h}\right)-\hat{p}_{\mathrm{ss}}\left(S_{m} \mid R_{h}\right)\right|}{p_{\mathrm{ss}}\left(S_{m} \mid R_{h}\right)} & \leq\left(\frac{1+\delta}{1-\delta}\right)^{M}-1 \\
& =2 M \delta+O\left(\delta^{2}\right) .
\end{aligned}
$$

Furthermore, since $p_{\mathrm{ss}}\left(S_{m} \mid R_{h}\right) \leq 1$, a looser bound for the element wise estimation error of $p_{\text {ss }}\left(S_{m} \mid R_{h}\right)$ can be given by $\left|p_{\mathrm{ss}}\left(S_{m} \mid R_{h}\right)-\hat{p}_{\mathrm{ss}}\left(S_{m} \mid R_{h}\right)\right| \leq((1+\delta) /(1-\delta))^{M}-$ $1 \approx 2 M \delta$, where the $O\left(\delta^{2}\right)$ term can be dropped for small $\delta$. Maximizing $\sum_{h=1}^{H} \mathbf{r}_{h} \hat{\mathbf{p}}_{s s}\left(R_{h}\right)^{T} \hat{\mathbf{q}}(\&)$ in (10) based on estimation leads to a pure rule $R_{h}$ (by Proposition 1) with estimated steady state utility that differs from the real steady state utility by no more than

$$
\begin{aligned}
& \left|\mathbf{p}_{\mathrm{ss}}\left(R_{h}\right)^{T} \mathbf{q}(\delta)-\hat{\mathbf{p}}_{\mathrm{ss}}\left(R_{h}\right)^{T} \hat{\mathbf{q}}(8)\right| \\
& \quad \leq \sum_{h=1}^{M}\left|p_{\mathrm{ss}}\left(S_{m} \mid R_{h}\right) Q\left(S_{m}\right)-\hat{p}_{\mathrm{ss}}\left(S_{m} \mid R_{h}\right) \hat{Q}\left(S_{m}\right)\right| \\
& \quad \leq \sum_{h=1}^{M}\left|p_{\mathrm{ss}}\left(S_{m} \mid R_{h}\right)-\hat{p}_{\mathrm{ss}}\left(S_{m} \mid R_{h}\right)\right| \max \left(Q\left(S_{m}\right), \hat{\mathrm{Q}}\left(S_{m}\right)\right) \\
& \quad \quad+p_{\mathrm{ss}}\left(S_{m} \mid R_{h}\right)\left|Q\left(S_{m}\right)-\hat{Q}\left(S_{m}\right)\right| \\
& \leq M U_{Q} \delta+2 M^{2} \delta \sigma \\
& =M \delta\left(U_{Q}+2 M \sigma\right) .
\end{aligned}
$$

Hence, the true optimal rule $R^{*}$ will have estimated average steady state utility with an error of $M \delta\left(U_{Q}+2 M \sigma\right)$. The 
(1) Initialize state transition count, mixed rule count, and utilities for each state.

For all states and actions $s, s^{\prime}, a$,

If there exists $R_{h} \in \mathcal{R}$ such that $R_{h}(s)=a$,

Set state transition count $C\left(s^{\prime}, s, a\right)=1$.

Else

Set state transition count $C\left(s^{\prime}, s, a\right)=0$.

Set rule count $c_{h}:=1$ for all $R_{h} \in \mathcal{R}$.

For all states $s \in \mathcal{S}$, set state utilities $Q^{(0)}(s):=0$.

Set state visit counts $\left(v_{1}, \ldots, v_{m}\right)=(0, \ldots, 0)$.

Set initial iteration $t:=0$.

Determine initial state $s_{0}$.

(2) Choose a rule.

Select mixed rule $R^{(t)}=\mathbf{r} \cdot \mathcal{R}$, where $\mathbf{r}=\left[\sqrt[M]{c_{1}}, \ldots, \sqrt[M]{c_{H}}\right]^{T} / \sum_{h=1}^{H} \sqrt[M]{c_{h}}$.

Calculate $a_{t}=R^{(t)}(s)$ for current state $s$.

(3) Update state transition probability and utility based on observed new state.

Process stream for given interval, and update time $t:=t+1$.

For new state $s_{t}=S_{h}$, measure utility $\widetilde{Q}$.

Set: $Q^{(t)}\left(S_{h}\right):=v_{h} Q^{(t-1)}\left(S_{h}\right) /\left(v_{h}+1\right)+\widetilde{Q} /\left(v_{h}+1\right)$.

Set: $v_{h}=v_{h}+1$.

Update: $C\left(s_{t}, s_{t-1}, R^{(t-1)}\left(s_{t-1}\right)\right):=C\left(s_{t}, s_{t-1}, R^{(t-1)}\left(s_{t-1}\right)\right)+1$.

For all $s, s^{\prime} \in \delta$, set: $p\left(s^{\prime} \mid s, a\right)=C\left(s^{\prime}, s, a\right) / \sum_{s^{\prime \prime} \in \delta} C\left(s^{\prime \prime}, s, a\right)$.

(4) Calculate utilities that would be achieved by each rule, and choose best pure rule.

Calculate steady-state state probabilities $\mathbf{p}_{\mathrm{ss}}\left(R_{h}\right)$ for pure rules.

Set $h^{*}:=\arg \max _{h \mid R_{h} \in \mathcal{R}} \mathbf{q}^{T} \mathbf{p}_{\mathrm{ss}}\left(R_{h}\right)$, where $\mathbf{q}=\left[Q^{(t)}\left(S_{1}\right), \ldots, Q^{(t)}\left(S_{M}\right)\right]^{T}$.

Update $c_{h^{*}}:=c_{h^{*}}+1$.

(5) Return to step 2.

Algorithm 1: (SPERO)

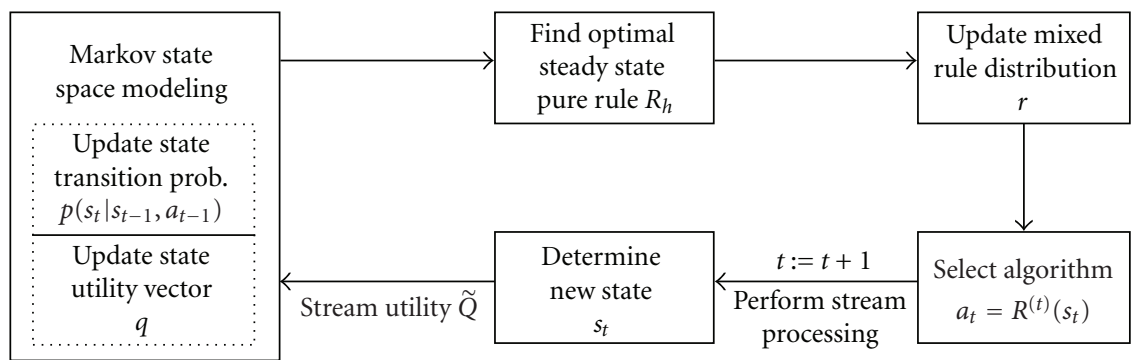

FIgURE 5: Flow diagram for updating parameters in Algorithm 1.

estimated rule $\widehat{R}^{*}$ will have at least the same estimated average utility of the true optimal rule and a true average utility within $M \delta\left(U_{Q}+2 M \sigma\right)$ of that value. Hence, combining the two maximum errors, we have the bound $2 M \delta\left(U_{Q}+2 M \sigma\right)$ for differences between the performances of the convergent rule and the optimal rule.

Corollary 1. In the worst case, the expected number of iterations required for SPERO to determine a pure rule that has average utility within $M \delta\left(U_{Q}+2 M \sigma\right)$ of the optimal pure rule with probability at least $(1-\varepsilon)(1-\eta)$ is $O\left(\max _{m=1, \ldots, M}\left(1 /\left(4 n \delta^{2}\right), v_{m}^{2} /\left(\varepsilon \sigma^{2}\right)\right)\right)$.

Proof. $\max _{m=1, \ldots, M}\left(1 /\left(4 n \delta^{2}\right), v_{m}^{2} /\left(\varepsilon \sigma^{2}\right)\right)$ is the greater value between the number of visits to each state required for $\operatorname{Pr}\left\{\left|Q\left(S_{m}\right)-\hat{Q}\left(S_{m}\right)\right| \geq \sigma\right\} \leq \varepsilon$, and the number of state transition occurrences required for $\operatorname{Pr}\left\{\left|\mathbf{P}_{i j}\left(R_{h}\right)-\widetilde{\mathbf{P}}_{i j}\left(R_{h}\right)\right| \geq\right.$ $\delta\} \leq \eta$. The number of iterations required to visit each state once is bounded below by the sojourn time of each state, which is, for recurrent states, a positive number $\tau$. Multiplying $\tau$ by the number of state visits required to meet the two Chebyshev bounds gives us the expected number of iterations required by SPERO.

Note that we use big-O notation, since the sojourn time $\tau$ for each recurrent state is finite, but this can also vary depending on the system dynamics and the convergent rule.

\section{Extensions of the Rules-Based Framework}

5.1. Evolving a New Rule from Existing Rules. Recall that SPERO determines the optimal rule out of a predefined set of rules. However, suppose that we lack the intuition to prescribe rules that perform well under any system state due 

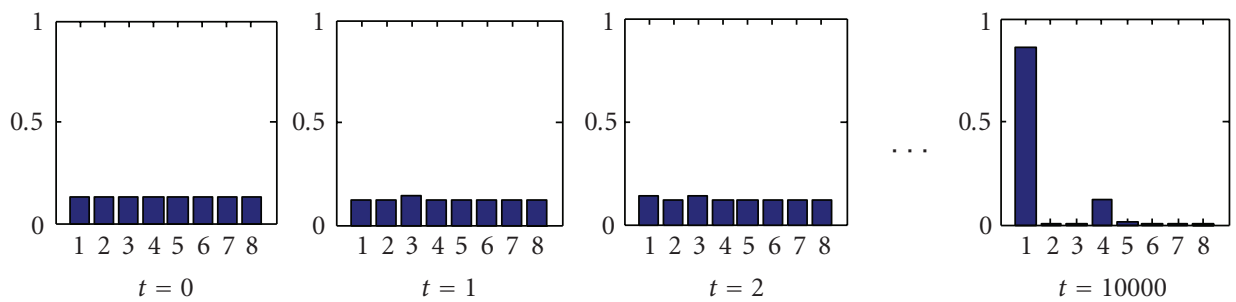

FIGURE 6: Rule distribution update in SPERO for 8 pure rules (see Section 6).

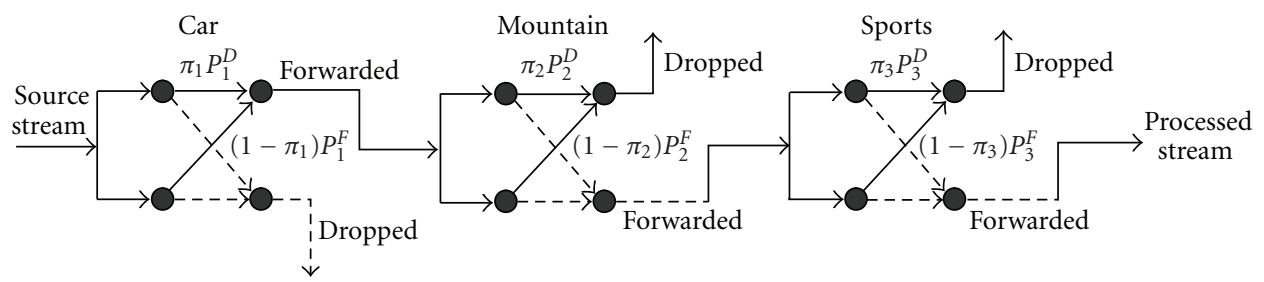

FIgURE 7: Chain of classifiers for car images that do not include mountains, nor are related to sports.

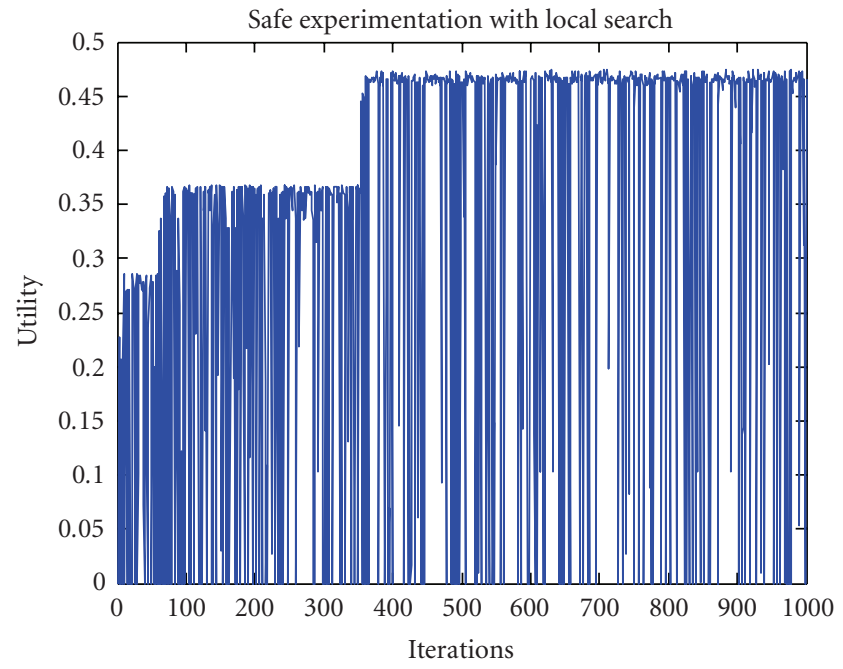

Figure 8: Convergence of safe experimentation.

to unknown stream dynamics. In this subsection, we propose a solution that evolves a new rule out of a set of existing rules.

Consider for each state $S_{m}$ a set of preferred algorithms $\mathcal{A}_{S_{m}}$, given by the algorithms that can be played in the state by the set of existing rules $\mathcal{R}$. Instead of changing the probability density of mixed rule $\mathbf{r} \cdot \mathcal{R}$ through reinforcing each existing rule, we propose a solution called Evolution From Existing Rules (EFER), which reinforces the probability of playing each preferred algorithm in each state based on its expected performance (utility) in the next time interval. Since EFER determines an algorithm for each state that may be prescribed by several different rules, the resulting scheme is not simply a mixed rule over the original set of pure rules $\mathcal{R}$, but rather an evolved rule over a larger set of pure rules $\mathcal{R}^{\prime}$.

Next, we present an interpretation on the evolved rule space. The rule space $\mathcal{R}^{\prime}$ can be interpreted by labeling each mixed rule $R$ over the original rule space $\mathcal{R}$ as an $M \times K$ matrix R, with entries $\mathbf{R}(m, k)=p\left(A_{k} \mid S_{m}\right)=\sum_{h=1}^{H} \mathbf{r}_{h}$. $\mathrm{I}\left(R_{h}\left(S_{m}\right)=A_{k}\right)$, and $\mathrm{I}()$ is the indicator function. Note that for pure rules $R_{h}$, exactly 1 entry in each row $m$ is 1 , and all other entries are 0 , and any mixed rule $\mathbf{r} \cdot \mathcal{R}$ lies in the convex hull of all pure rule matrices $\mathbf{R}_{1}, \mathbf{R}_{2}, \ldots, \mathbf{R}_{H}$ (See Figure 12 for a simple graphical representation.). An evolved rule $R^{\prime}$, on the other hand, is a mixed rule over a larger set $\mathcal{R}^{\prime} \supset \mathcal{R}$, which has the following necessary and sufficient condition: each row of rule $\mathbf{R}^{\prime}$ is in the convex hull of each rowof pure rule matrices $\mathbf{R}_{1}, \mathbf{R}_{2}, \ldots, \mathbf{R}_{H}$.

An important feature to note about EFER is that the evolved rule is not designed to maximize the steady state expected utility. SPERO can determine the steady state utility for each rule based on its estimated transition matrix. However, no such transition matrix exists for EFER, since, in the evolution of a new rule, there is no predefined rule to map each state to an algorithm, that is, no transition matrix for an evolving rule (until it converges). Hence, EFER focuses instead on finding the algorithm that gives the best expected utility during the next time interval (similar to best response play [43]). In the simulations section, we will discuss the performance tradeoffs between SPERO and EFER, where steady state optimization and best response optimization lead to different performance guarantees for stream processing.

5.2. A Decomposition Approach for Complex Sets of Rules. While using a larger state and rule space can improve the performance of the system, the complexity of finding the optimal rule in Solution 1 in Algorithm 1 increases significantly with the size of the state space, as it requires calculating the eigenvalues of $H$ different $M \times M$ matrices (one for each rule) during each time interval. Moreover, the convergence time to the optimal rule grows exponentially with the number of states $M$ in the worst case! Hence, for a finite number of time intervals, a larger state space can even 
(1) Initialize state transition count, prescribed algorithm probabilities, and utilities for each state.

For all states and actions $s, s^{\prime}$, $a$, set state transition count $C\left(s^{\prime}, s, a\right)=1$.

Initialize algorithm probability count

$c\left(S_{m}, A_{k}\right):=\sum_{h=1}^{H} \mathrm{I}\left(R_{h}\left(S_{m}\right)=A\right)$ foreach $S_{m}$ and $A_{k}$.

For all states $s \in 8$, set state utilities $Q^{(0)}(s):=0$.

Set state visit counts $\left(v_{1}, \ldots, v_{m}\right)=(0, \ldots, 0)$.

Set initial iteration $t:=0$.

Determine initial state $s_{0}$.

(2) Choose an algorithm.

Select algorithm $A_{k}$ with probability $p\left(A_{k}\right)=c\left(s_{t}, A_{k}\right) / \sum_{\kappa=1}^{K} c\left(s_{t}, A_{\kappa}\right)$.

(3) Update state transition probability and utility based on observed new state.

Process stream for given interval, and update time $t:=t+1$.

For new state $s_{t}=S_{m}$, measure utility $\widetilde{Q}$.

Set: $Q^{(t)}\left(S_{h}\right):=v_{h} Q^{(t-1)}\left(S_{h}\right) /\left(v_{h}+1\right)+\widetilde{Q} /\left(v_{h}+1\right)$.

Set: $v_{h}=v_{h}+1$.

Update: $C\left(s_{t}, s_{t-1}, R^{(t-1)}\left(s_{t-1}\right)\right):=C\left(s_{t}, s_{t-1}, R^{(t-1)}\left(s_{t-1}\right)\right)+1$.

For all $s, s^{\prime} \in \delta$, set: $p\left(s^{\prime} \mid s, a\right)=C\left(s^{\prime}, s, a\right) / \sum_{s^{\prime \prime} \in \delta} C\left(s^{\prime \prime}, s, a\right)$.

(4) Calculate the expected utility in the next time interval, and increment frequency of the best algorithm in the last state. If $Q^{(t)}\left(S_{m}\right)=\max \left\{Q^{(t)}\left(S_{\eta}\right) \mid \eta=1, \ldots, H\right\}$,

Set $k^{*}:=\arg \max _{k \mid A_{k} \in \mathcal{R}} \sum_{k=1}^{K} \sum_{s^{\prime} \in s} p\left(s^{\prime} \mid s, A_{k}\right) Q\left(s^{\prime}\right)$, where :

$\mathbf{q}=\left[Q\left(S_{1}\right), \ldots, Q\left(S_{M}\right)\right]^{T}$.

Increment $c_{h^{*}}:=c_{h^{*}}+1$.

(5) Return to step 2 and repeat.

Algorithm 2: (EFER)

perform more poorly than a smaller state space (as we will show in our simulations).

To overcome the complexity issue, we propose a decomposition method that omits a subset of rules in order to reduce a large rule space into a collection of simple rules that can be decided autonomously by each classifier site. We define the decomposition methods below.

Definition 4. Consider a centralized state space model $\&$ for a system of $n$ different sites. $\delta$ is said to be decomposable if $\delta=$ $\delta_{1} \times s_{2} \times \cdots \times s_{n}$, where $\delta_{i}$ is a local state space model at site i. Likewise, $\delta$ is partially decomposable if $\delta=\delta_{1} \times s_{2} \times \cdots \times$ $\delta_{n} \times \delta^{\prime}$, where $S^{\prime}$ is a shared state space model that is contained in all local models. In other words, all local state space models are of the form $\delta_{i} \times{\delta^{\prime}}^{\prime}$. Similarly, an algorithm space model is said to be decomposable if $\mathcal{A}=\mathcal{A}_{1} \times \mathcal{A}_{2} \times \cdots \times \mathcal{A}_{n}$, where the algorithm space $\mathcal{A}_{i}$ is the set of algorithms that can be used to reconfigure system parameters at site $i$.

Definition 5. A decomposable rule space model $\mathcal{R}=\mathcal{R}_{1} \times \mathcal{R}_{2} \times$ $\cdots \times \mathcal{R}_{n}$ is given over a decomposablealgorithm space model $\mathcal{A}=\mathcal{A}_{1} \times \mathcal{A}_{2} \times \cdots \times \mathcal{A}_{n}$ and a partially decomposable state space model $\delta=s_{1} \times s_{2} \times \cdots \times s_{n} \times \delta^{\prime}$, where each local rule in $\mathcal{R}_{i}$ maps a local state in $\delta_{i} \times \delta^{\prime}$ to a local algorithm in $\mathcal{A}_{i}$.

Note that, in a decomposed rule space model, each site has its own set of local rules and algorithms that it plays independently based on partial information (or a state space model using partial information) about the entire system. The notion of partial information has several strong implications. For example, a centralized rule space is not always decomposable, even when it is played over a decomposable algorithm and state space (See Example 1.). Hence, there always exist centralized rules that cannot be simulated by a decomposed approach. Furthermore, when the local state space models are not identical between each classifier, the classifiers converge to a Nash equilibrium [43] when running SPERO locally and independently, even when their payoffs are identical. While proof of convergence is a straightforward extension of Proposition 3, it is difficult to prove conditions under which the convergence point is optimal or suboptimal, since multiple Nash equilibria may exist [43]. In general, the convergent rule depends highly on the initial rules used in SPERO (see Example 2). However, as we demonstrate in Example 2, the probability of converging to a suboptimal rule is also correlated with its efficiency, such that poor equilibria are reached with low probability.

Example 1 (when a rule cannot be decomposed). Consider a centralized state space given by 4 states consisting of quantized local utilities of a 2 classifier system. Each classifier has a "bad" state $S_{1, i}$ corresponding to $\widetilde{Q}_{i}\left(P_{i}^{F}\right)<Q_{\text {thresh }}$, and a "good" state $S_{2, i}$ corresponding to $\widetilde{Q}_{i}\left(P_{i}^{F}\right) \geq Q_{\text {thresh. }}$. Each classifier can perform a local algorithm $A_{1, i}$ given by randomly choosing a new configuration (experimentation) or performing a local search $A_{2, i}$ around the last configuration, and to memorize the new configuration if it outperforms the old (See [13] for details.). A centralized rule space can consist of all rules $R: \wp_{1} \times \wp_{2} \rightarrow \mathcal{A}_{1} \times \mathcal{A}_{2}$, while a localized rule space can only consist of rules of the form $R=\left(R^{(1)}, R^{(2)}\right)$, 
where $R^{(1)}: \wp_{1} \rightarrow \mathcal{A}_{1}$, and $R^{(2)}: \wp_{2} \rightarrow \mathcal{A}_{2}$. A decomposable rule is for each classifier to use experimentation in state $S_{1, i}$ and local search in state $S_{2, i}$. A nondecomposable rule is for each classifier to use experimentation in all states, unless both classifiers are in state $S_{2, i}$. As can be seen, to use nondecomposable rules, each classifier needs information about the states of both classifiers.

Example 2 (convergence to a suboptimal equilibrium). Consider a simple scenario involving two classifiers $i=$ 1,2, and two algorithms for each classifier, $A_{1, i}$ and $A_{2, i}$. The centralized model contains four states given by the combinations of algorithms used in the previous time interval. Suppose that when both classifiers perform action $A_{1, i}$, the utility of the system in the following time interval is 2 . When both classifiers perform action $A_{2, i}$, the utility of the system is 1 . Otherwise, the utility is 0 . In the local model, each classifier measures only two states, where each state is given by the algorithm that it performed during the last interval, that is, $S_{1, i}=A_{1, i}, S_{2, i}=A_{2, i}$. Suppose that during the first 100 iterations, the following actions happen to be played: $\left(A_{1,1}, A_{1,2}\right)$ with probability 1/100, $\left(A_{2,1}, A_{1,2}\right)$ with probability $9 / 100,\left(A_{1,1}, A_{2,2}\right)$ with probability $9 / 100$, and $\left(A_{2,1}, A_{2,2}\right)$ with probability $81 / 100$. (Note that these classifiers are probabilistically choosing algorithms independently.) Then for each classifier, the estimated utility of using algorithm $A_{1, i}$ is $1 / 10 * 2=1 / 5$, while the utility of using algorithm $A_{2, i}$ is $9 / 10$. Each classifier will thus continue to reinforce its own algorithm $A_{2, i}$, leading to a convergent suboptimal rule of using $\left(A_{2,1}, A_{2,2}\right)$ with probability 1 (unless the state/action $\left(A_{1,1}, A_{1,2}\right)$ is played a significant fraction of time to update the local utilities). Note that $\left(A_{2,1}, A_{2,2}\right)$ is a Nash equilibrium as well as the optimal $\left(A_{1,1}, A_{1,2}\right)$.

Note that while, in Example 2, suboptimal convergence is possible, the likelihood of suboptimal convergence to $\left(A_{2,1}, A_{2,2}\right)$ is dependent on the utilities achieved in the two Nash equilibria. The greater the difference between the utilities of the Nash equilibria, the more unlikely the distributed approach is to converge to a suboptimal rule. For example, suppose that $Q\left(A_{1,1}, A_{1,2}\right)=\alpha>1$, and $Q\left(A_{2,1}, A_{2,2}\right)=1$, and the utility is zero otherwise. Then algorithm $A_{2, i}$ must be played with probability of at least $1-1 /(\alpha+1)$ in order for both classifiers to reinforce the suboptimal combination of algorithms $\left(A_{2,1}, A_{2,2}\right)$. Hence, for large $\alpha$, suboptimal convergence is unlikely to occur unless initial conditions are heavily weighted towards $\left(A_{2,1}, A_{2,2}\right)$.

\section{Simulation Results}

6.1. Application: Classification of TV Video Data. Our proposed algorithm is tested using classifiers and videos provided by IBM's TRECVID 2007 project [44]. By extracting features such as color histogram, color correlogram, and co-occurrence texture, the classifiers are trained to detect high-level features, such as whether the video shot takes place outdoors, or in an office building, or whether there is an animal or a car in the video. The classifiers are SVMbased and can therefore dynamically set detection thresholds for the output scores for each image without changing the underlying implementation. We chose this dataset due to the wide range of high-level features detected, which best models classifiers trained across different sites. We chose to construct a chain out of classifiers to detect images that contained cars but did not include mountains and were not sports related, as this includes a sizable fraction of images from the total set (113 images from a total of 18000) and also requires heavy filtering of images at each classifier. The arrangement of classifiers is shown in Figure 7. The resource available across each classifier constitutes approximately 1/10 of the resource required by the classifiers to process the true fraction of positive data. The application delay sensitivity is set to a DPF $\varphi=50$.

6.2. Motivation for Using Rules: an Experimentation Algorithm. To motivate the need for rules, consider first the safe experimentation algorithm introduced in our prior work [13]. We applied the algorithm to the existing dataset and discovered that the algorithm converged to the optimal performance (i.e., optimal fusion of decision thresholds for the classifier chain) when excess processing resources were available and delay sensitivity was low (See Table 2). Furthermore, compared to the optimal fusion of classifier scores without considering resource constraints, our algorithm boosted the detection rate by an order of magnitude while reducing the processing delay when resource constraints were scarce (only about 10\% of the stream could be processed from end-to-end!), and delay sensitivity was high. This was achieved by jointly choosing the operating points based on both the load at downstream classifiers as well as the overall classification performance/cost, thus leading to intelligent load shedding of low-confidence data. However, note from Figure 8 that convergence of the experimentation algorithm is slow and requires several hundred iterations before reaching the optimal configuration. Consequently, if the stream characteristics (e.g., a priori probability) change significantly within a hundred iterations, the performance of this algorithm will not be able to adapt quickly enough to optimize the system. In fact, as we will show later, the performance can be significantly improved by using a decomposed rule space, where each classifier individually chooses from a small set of algorithms.

6.3. State Space, Algorithms, and Rules Used in Simulations. In our experiments, we use the following state space quantizations and algorithms listed hereinafter.

(i) State Space. In our experiments, we associate four states $S_{1}, S_{2}, S_{3}, S_{4}$ with different levels of "minimum" utility given by $0,0.1,0.2$, and 0.3 , respectively. Note that the utilities are small due to the delay penalty factor as well as the low a priori probability of the class of interest. The "minimum" utility levels merely determine bounds for being in each state and are not regarded as the average utilities estimated in each 
TABLE 2: Detection and false alarm tradeoff for the entire chain after global convergence of algorithms

\begin{tabular}{llll}
\hline & Safe Exp & Safe Exp with local search & $\begin{array}{c}\text { Optimal classifier configuration } \\
\text { with "random load shedding" }\end{array}$ \\
\hline High resources (pd, pf) & $0.8053,0.3376$ & $0.8053,0.3291$ & $0.8053,0.3291$ \\
Low resources (pd, pf) & $0.1062,0.0024$ & $0.1062,0.0024$ & $0.0060,0.0025$ \\
Low resources (delay [secs], $\operatorname{Pr}\{D>5\})$ & $3.98,0.2847$ & $3.98,0.2847$ & $6.06,0.4382$ \\
\hline
\end{tabular}

state. Furthermore, the state space can be divided into local states for each classifier that capture different ranges of local utilities. We used a low state 0 and a high state 0.1 for the local utilities of each classifier.

(ii) Algorithms. The algorithm space consists of 4 algorithms modified from the solution proposed in [13]. Algorithm $A_{1}$ randomly chooses a new configuration for the classifier. $A_{2}$ samples a random configuration near its current best (or baseline) configuration, and if the utility increases with the new configuration, set the new baseline configuration to the new configuration. Additionally, we use two algorithms $A_{3}, A_{4}$ to perform random experimentation in low $P^{F}$ (below the equal error rate configuration) and high $P^{F}$ (above the equal error rate configuration) regions of each classifier.

We will compare 3 different types of rules-based approaches.

(i) The first approach (Experimentation) involves a single fixed (but fairly efficient) rule, which performs algorithm $A_{1}$ when the system utility is below a threshold (0.2), and algorithm $A_{2}$ otherwise. This approach is very similar to the algorithm proposed in [13]. This approach has the lowest complexity of all the approaches.

(ii) The second approach (Small Rule Space) uses a state space consisting of the 4 different levels of minimum utility and a centralized algorithm that allocates identically to each and every classifier, one of the 4 algorithms. To map each state to an algorithm, 8 heuristic rules are used. SPERO is used to determine the optimal steady state rule.

(iii) The third approach (Distributed/Large Rule Space) uses a large state space with 4 levels of utility as well as 2 levels of local utilities for each classifier, totaling 32 states. Due to the high complexity and long convergence time of the centralized approach, we use decomposition by configuring each classifier independently using the 4 algorithms, leading to a total of $4^{3}=64$ possible algorithms. Finally, we consider 512 decomposable pure rules, where the rule space is a cross product between 8 local rules at each classifier. Note that the actual rule space at each classifier is similar to the second approach (8 states, 4 algorithms, 8 rules), although the combined centralized rule space is huge. SPERO is used at each classifier independently to learn the optimal local rule.
6.4. Comparison of Algorithms under Different Levels of Dynamics. In Figure 9, we display the average utilities achieved over the first 10000 time intervals of SPERO under different rates of change (given in Section 6.1). We discovered that the average performance of the first approach (experimentation) decreases as the rate of change increases, since changing stream characteristics requires the experimentation approach to randomly sample different points frequently when the utility level drops below the fixed threshold. In a highly dynamic case (e.g., rate of change equal to 12), the experimentation approach obtains an average utility that outperformed the small rule space by approximately $20 \%$. The poor performance can be attributed to the poor choice of rules, where, out of the 8 rules, the rule that corresponds to the first approach actually outperforms all other rules. However, because SPERO performs random selection out of all 8 rules and requires many iterations to converge, the average performance is poorer during the first 10000 iterations. Finally, we discovered that in the third approach (large rules-space), which we implemented in a distributed fashion across classifiers due to its high complexity, the rule space contained a convergent rule that significantly outperformed the optimal rule in the first two approaches. The average utility for the first 10000 iterations was about $27 \%$ higher than the experimentation approach. This is because the decomposed rule enables each classifier to model better the dynamics in its own local environment, which has a greater effect on its individual performance and delay.

On the other hand, for static or near static environments, we discovered that approaches 2 and 3 usually performed worse than experimentation. This is because, in slowly timevarying environments, the optimal rule in both the small and large rule spaces is in fact the experimentation rule. However, because of their slower learning rates, approaches 2 and 3 tend to perform more poorly during the first 10000 iterations while trying to discover (and reinforce) the experimentation rule.

To provide better intuition about the utilities achieved by each approach, we constructed a table of the confusion matrices and delays (see Table 3 and Figure 10) under a very dynamic environment (the volume of video images in each of the 8 possible intersection of classes varied by 12 per every interval, with each class size averaging 2500 images). Note that the "labeled no" class refers to data that has been dropped (i.e., misses and true negatives) and is significantly greater than the detected images due to system load shedding. The misses can be attributed to both classifier inaccuracy as well as discarding of low-confidence data to 
ensure that correctly classified data is received with low delay. From Table 3, it can be seen that the experimentation approach performs very poorly, since whenever it obtains a configuration with high utility, the stream dynamics change within the next few time intervals, forcing the solution to perform random experimentation again. On the other hand, the small rule space had a better confusion matrix, but the utility suffered from the long end-to-end processing delay and high-delay variance. This is due to periodically choosing suboptimal rules that operate at high false alarm regions even when the APP is high. Hence, the experimentation approach achieved a higher delay-sensitive utility than the small rule space. Finally, the large/distributed rule space provided the best performance as well as the lowest average delay and delay variance. As indicated by Table 4, each classifier converges toward a different local rule that is highly dependent on its accuracy and resource constraints. (In Table 4, rule 2 corresponds to approach 1, while other rules are mixtures of local search and random configurations in low and high false alarm regions.) Importantly, Table 4 shows that by decomposing 512 rules into 8 local rules at each classifier, SPERO converges quickly to a rule that performs well under the given dynamics.

6.5. Evaluation of the Markov Assumption for Algorithms. An important consideration is whether system dynamics are accurately modeled by the algorithmic Markov process given in Definition 1. To determine the sufficiency of information captured by the last state, we calculated the state transition probabilities for each algorithm conditioned on only the last state, versus the state transition probabilities conditioned on the last 2 states. The similarity between distributions obtained based on the last state, and the last two states, was evaluated for each algorithm using the average absolute difference between the estimated state transition probabilities. In other words, we evaluated a distance metric $(1 / M) \sum_{s_{t} \in \delta}\left|\operatorname{Pr}\left\{s_{t} \mid s_{t-1}, a_{t-1}\right\}-\operatorname{Pr}\left\{s_{t} \mid s_{t-1}, a_{t-1}, s_{t-2}\right\}\right|$ for each $a_{t-1}$ and $s_{t-2}$. We discovered that, for the centralized state space partitioned into 4 bins based on utilities, the (first-order) Markov model and the second-order Markov model had transition probabilities that differed element wise by no more than 0.04 . This shows that the first-order Markov model is sufficient in capturing most of the information about the past two states, which provides higher confidence in the accuracy of Markov modeling for algorithms for the distributed classification system.

6.6. Evolution of a New Rule. In this section, we used EFER to evolve a new rule from the large/distributed rule space. We compared the performance of our evolved rule with the convergent distributed rule in the previous section and discovered that the average performance of the evolved rule was about $10 \%$ worse than that of the best prescribed rule in the large/distributed rule space. However, as shown in Figure 11 for a highly dynamic environment, EFER provides smaller utility fluctuations and guarantees a better minimum utility with high probability.

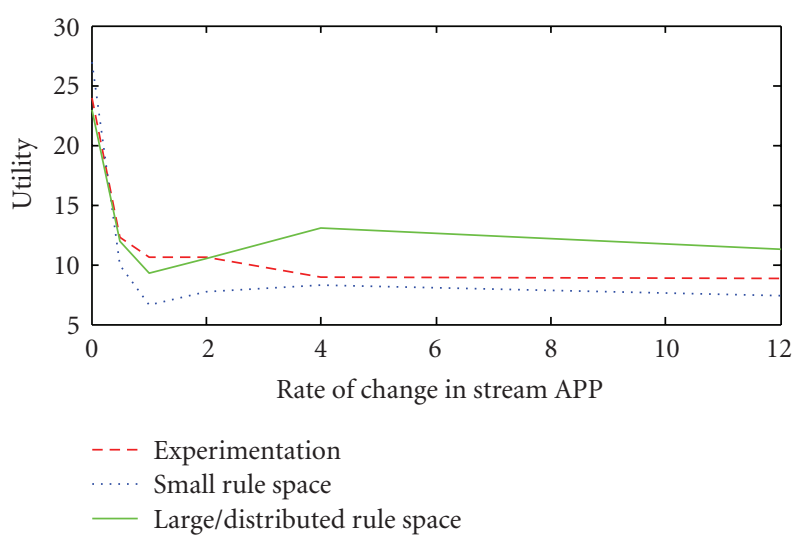

FIGURE 9: Comparison of utilities $(\times 0.01)$ achieved by different rule spaces under different levels of dynamics.

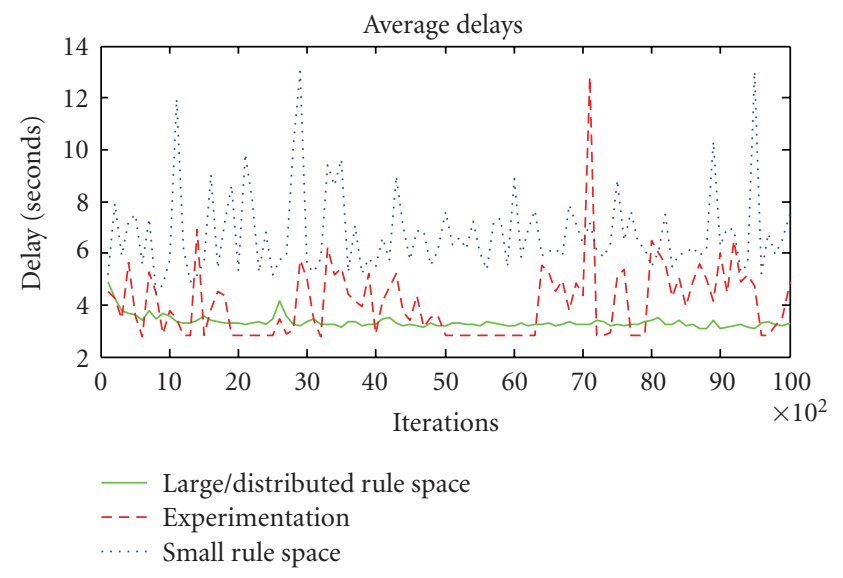

FIGURE 10: Comparison of delays for the 3 approaches. Each point is an average delay over 100 intervals.

This phenomenon can be explained by how SPERO and EFER updates rules. Recall that, in SPERO, the mixed rule was updated by reinforcing the pure rule with the highest steady state performance. Such a rule may perform well in certain states, but poorly in other states, since transients are ignored in this approach for the sake of maximizing the average performance. However, the evolved rule, which chooses an algorithm based on the expected performance in the next time interval, is more likely to discover a rule that performs well in each state, although not necessarily the rule that provides the optimal steady state performance.

Finally, note that the complexity of EFER is much less than SPERO, since EFER is not required to compute the eigenvalues for every pure rule matrix. Rather, it performs a single matrix-vector multiplication per algorithm and chooses the best algorithm for the next time interval. From our experiments, the running time for determining the rule to reinforce during each iteration in SPERO was approximately 14.0 milliseconds, while the running time to determine the algorithm to reinforce in EFER was only 5.1 milliseconds. The savings become even more significant when the number of rules in SPERO becomes larger. 
TABLE 3: Average confusion matrices per time interval for a dynamic stream of images.

\begin{tabular}{lcccccc}
\hline \multirow{2}{*}{ Approach } & \multicolumn{2}{c}{ Experimentation } & \multicolumn{2}{c}{ Small rule space } & \multicolumn{2}{c}{ Large rule space } \\
& Lbled Yes & Lbled No & Lbled Yes & Lbled No & Lbled Yes & Lbled No \\
\hline Yes & 740 & 1003 & 751 & 587 & 885 & 453 \\
No & 815 & 11382 & 2220 & 9349 & 710 & 10860 \\
\hline Average delay & \multicolumn{3}{c}{3.98 secs. } & \multicolumn{3}{c}{6.50 secs. } \\
\hline
\end{tabular}

TABLE 4: Probabilities of using local rules by each classifier for the distributed large rule space.

\begin{tabular}{lllllllll}
\hline Classifier & Rule 1 & Rule 2 & Rule 3 & Rule 4 & Rule 5 & Rule 6 & Rule 7 & Rule 8 \\
\hline 1 & $0.05 \%$ & $\mathbf{8 8 . 6} \%$ & $0.01 \%$ & $0.49 \%$ & $8.71 \%$ & $0.96 \%$ & $0.02 \%$ & $1.11 \%$ \\
2 & $0.64 \%$ & $0.06 \%$ & $4.29 \%$ & $3.80 \%$ & $0.07 \%$ & $\mathbf{9 1 . 0} \%$ & $0.01 \%$ & $0.18 \%$ \\
3 & $\mathbf{8 5 . 6} \%$ & $0.01 \%$ & $0.32 \%$ & $12.0 \%$ & $1.69 \%$ & $0.12 \%$ & $0.06 \%$ & $0.28 \%$ \\
\hline
\end{tabular}

TABLE 5: Average distance between a first-order and second-order Markov model.

\begin{tabular}{ll}
\hline $\begin{array}{l}\text { State visited 2 } \\
\text { intervals ago }\end{array}$ & $\begin{array}{l}\text { Avg. absolute distance compared to } \\
\text { 1st-order Markov model }\end{array}$ \\
\hline 1 & 0.0032 \\
2 & 0.0373 \\
3 & 0.0353 \\
4 & 0.0362 \\
\hline
\end{tabular}

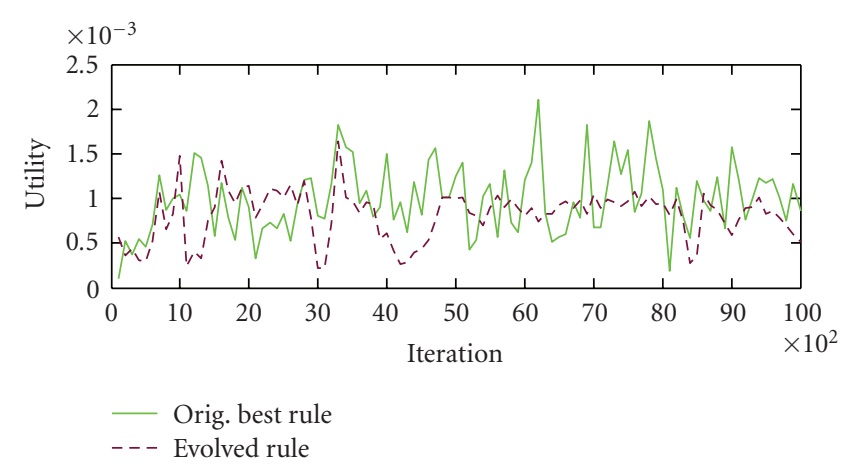

Figure 11: Comparison of utility achieved by the best rule in the original space, and the evolved rule.

\section{Conclusions}

In this paper, we proposed a rules-based framework for reconfiguring distributed classifiers for a delay-sensitive stream mining application with dynamic stream characteristics. By gathering information locally at each classifier and estimating local utility metrics, the framework employs rules based on models of the global system utility and transition probabilities between different states. We showed that the optimal rule can be chosen from a set of prescribed rules while accurately measuring parameters related to stream dynamics. Furthermore, we proposed a decomposition approach for reducing the complexity of the framework. Finally, we proposed a method to evolve a new rule based on prescribed rules. Using a chain of speech classifiers, we validated that large gains could be achieved by the proposed rules-based framework.

Note that while we used the classifier chain configuration problem as a key application to show the advantages of using rules to choose algorithms, the rules-based framework is not specific to the distributed stream mining problem and can be applied to various other dynamic and informationally distributed systems. Importantly, when system dynamics are unknown and intuition is insufficient for choosing the best algorithm to use for reconfiguration, the proposed methodology enables the system to adapt by learning the best algorithms to deploy under different system conditions.

The proposed framework is the first to capture a challenging problem described by distributed information, severe restrictions on information exchange, resource constraints, and dynamics. We see as a major avenue for future work, improving its application toward challenges that arise in other areas of research, such as autonomic computing and intelligent distributed systems. While not all of the above challenges may be present in a specific problem, questions regarding what type of distributed algorithms to use as part of the rules-based approach, and furthermore, how to best quantize these algorithms over the space of all algorithms (if such is possible), can lead to major breakthroughs for realtime systems and applications. A theoretical area of interest is also how to best combine different algorithms and make use of their properties to improve the overall performance.

\section{Appendix}

\section{Proof of Convergence of SPERO}

Proposition 3. For an algorithmic Markov system, SPERO converges to the optimal rule in steady state.

Proof. Note that the average utility in each state $Q\left(S_{m}\right)$, and the unknown state transition probabilities, must be perfectly estimated for each rule and state to determine the optimal rule. Since performing each state transition infinitely many times when $t \rightarrow \infty$ implies that each state is visited (and hence the utility is measured) infinitely many times, we need only prove perfect estimation for state transitions. 

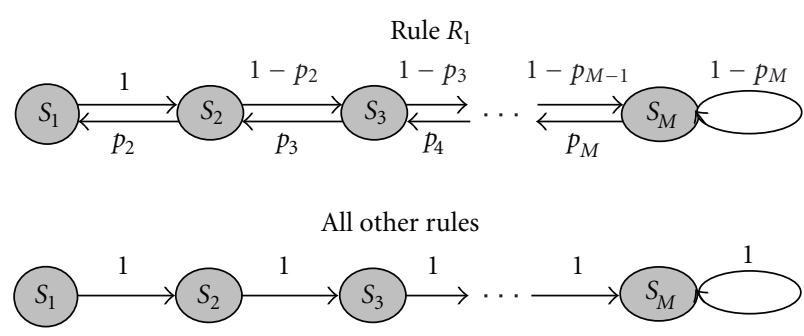

Figure 12: Worst case Markov chain for updating $Q\left(S_{1}\right)$, random walk on a line.

We use the worst-case lower bound for the number of times each rule is played in each state to prove that each feasible state transition occurs infinitely many times as $t \rightarrow$ $\infty$. Consider the Markov chain given in Figure 12, where, for a pure rule $R_{1}$, there exists a random walk from state $S_{m}$ to $S_{m+1}$ and $S_{m-1}$ with nonzero probabilities (except for $S_{1}$ and $\left.S_{M}\right)$. For all other pure rules, algorithms are chosen such that state transitions always lead from $S_{m}$ to $S_{m+1}$, until the state reaches $S_{M}$. Furthermore, we assume the relation $Q\left(S_{1}\right)<Q\left(S_{2}\right)<\cdots<Q\left(S_{M}\right)$, such that the solution reinforces rules leading away from $S_{1}$. This is the worst case scenario for updating the transition probabilities of $S_{1}$.

Let $H$ be the total number of pure rules. Suppose that at time $t$, all other rules have been reinforced a total of $t$ times, but $R_{1}$ has not been reinforced, that is, $c_{1}=1$. The worst case probability of playing $R_{1}$ at time $t$ is if all other rules have been reinforced equally, that is, $c_{2}=c_{3}=\cdots=c_{H}=$ $t /(H-1)$. In this case, rule $R_{1}$ is played with probability $\mathbf{r}_{1}=$ $1 /(1+(H-1) \sqrt[M]{t /(H-1)})$. The probability of transitioning from $S_{m}$ to $S_{1}$ in $M-1$ steps, and playing rule $R_{1}$ in state $S_{1}$ (hence playing $R_{1} M$ consecutive times), can be bounded by

$$
\begin{aligned}
& p\left(S_{M} \longrightarrow S_{1}, R_{1} \mid t\right) \\
& =\mathbf{r}_{1}(t) \cdot p_{M} \cdot \mathbf{r}_{1}(t+1) \cdot p_{M-1} \cdots \mathbf{r}_{1}(t+M-2) p_{2} \cdot \mathbf{r}_{1} \\
& >\prod_{m=2}^{M} p_{m} \prod_{m=0}^{M-1} \cdot \frac{1}{1+(H-1) \sqrt[M]{(t+m) /(H-1)}} \\
& \left.>C \cdot\left(\frac{1}{1+(H-1) \sqrt[M]{(t+M-1) /(H-1)}}\right)^{M}\right)^{M} \\
& >C \cdot\left(\frac{1}{2(H-1) \sqrt[M]{(t+M-1) /(H-1)}}\right. \\
& =C \cdot\left(\frac{1}{2(H-1)}\right)^{M} \frac{H-1}{t+M-1} \\
& >C C^{\prime} \frac{1}{t+M},
\end{aligned}
$$

where $C$ and $C^{\prime}$ are constants, and the inequality in the fourth line is due to the fact that $(H-1) \sqrt[M]{(t+m) /(H-1)}>$ 1. Since from any other starting state transitioning to $S_{1}$ in less than $M$ steps has higher probability than (A.1), we can also bound the average number of plays of rule $R_{1}$ in $S_{1}$ for every $M$ time intervals by (A.1). Likewise, the average number of visits to any other state (e.g., $S_{2}$ ) starting from any initial state, times the probability of using any rule in the final state, is also bounded below by (A.1).

Thus, the total number of updates for transition probability pairs for any state $S_{m}$ and rule $R_{h}$ as $t \rightarrow \infty$ can be bounded by

$$
\begin{aligned}
\lim _{t \rightarrow \infty} E\left[N_{S_{m}}(t)\right] & >\sum_{\hat{t}=0}^{\infty} \frac{C^{\prime}}{\hat{t} M+M} \\
& =C^{\prime}\left(\frac{1}{M}+\frac{1}{2 M}+\frac{1}{3 M}+\cdots\right)=\infty,
\end{aligned}
$$

where the right-hand side is given by recalculating the final inequality of (A.1) every $M$ time intervals, starting with $t=$ 0 .

\section{References}

[1] M. A. Shah, J. M. Hellerstein, S. Chandrasekaran, and M. J. Franklin, "Flux: an adaptive partitioning operator for continuous query systems," in Proceedings of the International Conference on Data Engineering (ICDE '03), pp. 25-36, March 2003.

[2] C. Olston, J. Jiang, and J. Widom, "Adaptive filters for continuous queries over distributed data streams," in Proceedings of the 22nd ACM SIGMOD International Conference on Management of Data (SIGMOD '03), pp. 563-574, San Diego, Calif, USA, June 2003.

[3] L. Amini, H. Andrade, F. Eskesen, et al., "The stream processing core," Technical Report RSC 23798, November 2005.

[4] D. Turaga, O. Verscheure, U. Chaudhari, and L. Amini, "Resource management for chained binary classifiers," in Proceedings of the Workshop on Tackling Computer Systems Problems with Machine Learning Techniques (SysML '06), 2006.

[5] Y. Schapire, "A brief introduction to boosting," in Proceedings of International Conference on Algorithmic Learning Theory, 1999.

[6] Y. Xing, S. Zdonik, and J.-H. Hwang, "Dynamic load distribution in the borealis stream processor," in Proceedings of the 21st International Conference on Data Engineering (ICDE '05), pp. 791-802, Tokyo, Japan, April 2005.

[7] M. Cherniack, H. Balakrishnan, M. Balazinska, et al., "Scalable distributed stream processing," in Proceedings of Conference on Innovative Data Systems Research (CIDR '03), Asilomar, Calif, USA, January 2003.

[8] A. Garg, V. Pavlović, and T. S. Huang, "Bayesian networks as ensemble of classifiers," in Proceedings of the International Conference on Pattern Recognition (ICPR '02), pp. 779-784, 2002.

[9] M. Balazinska, H. Balakrishnan, S. Madden, and M. Stonebraker, "Fault-tolerance in the borealis distributed stream processing system," in Proceedings of the ACM SIGMOD International Conference on Management of Data (SIGMOD '05), pp. 13-24, Baltimore, Md, USA, June 2005.

[10] R. Lienhart, L. Liang, and A. Kuranov, "A detector tree for boosted classifiers for real-time object detection and tracking," in Proceedings of the International Conference on Multimedia and Expo (ICME'03), 2003.

[11] D. S. Turaga, O. Verscheure, U. V. Chaudhari, and L. D. Amini, "Resource management for networked classifiers in 
distributed stream mining systems," in Proceedings of the IEEE International Conference on Data Mining (ICDM '07), pp. 1102-1107, December 2006.

[12] F. Douglis, M. Branson, K. Hildrum, B. Rong, and F. Ye, "Multi-site cooperative data stream analysis," ACM SIGOPS Operating Systems Review, vol. 40, no. 3, pp. 31-37, 2006.

[13] B. Foo and M. van der Schaar, "Distributed classifier chain optimization for real-time multimedia stream mining systems," in Multimedia Content Access: Algorithms and Systems II, vol. 6820 of Proceedings of SPIE, San Jose, Calif, USA, January 2008.

[14] S. Merugu and J. Ghosh, "Privacy-preserving distributed clustering using generative models," in Proceedings International Conference on Data Mining (ICDM '03), 2003.

[15] F. Fu, D. S. Turaga, O. Verscheure, M. van der Schaar, and L. Amini, "Configuring competing classifier chains in distributed stream mining systems," IEEE Journal on Selected Topics in Signal Processing, vol. 1, no. 4, pp. 548-563, 2007.

[16] E. M. Rounds, "A combined nonparametric approach to feature selection and binary decision tree design," Pattern Recognition, vol. 12, no. 5, pp. 313-317, 1980.

[17] N. Tatbul, "QoS-driven load shedding on data streams," in XML-Based Data Management and Multimedia Engineering, 2002.

[18] B. Babcock, S. Babu, M. Datar, and R. Motwani, "Chain: operator scheduling for memory minimization in data stream systems," in Proceedings of the 22nd ACM SIGMOD International Conference on Management of Data (SIGMOD '03), pp. 253-264, San Diego, Calif, USA, June 2003.

[19] N. Tatbul, U. Cetintemel, S. Zdonik, M. Cherniack, and M. Stonebraker, "Load shedding in a data stream manager," in Proceedings of the 29th International Conference on Very Large Databases (VLDB'03), September 2003.

[20] B. Babcock, M. Datar, and R. Motwani, "Cost-efficient mining techniques for data streams," in Proceedings of the Workshop on Management and Processing of Data Streams (MDPS '03), 2003.

[21] N. Tatbul and S. Zdonik, "Dealing with overload in distributed stream processing systems," in Proceedings of the IEEE International Workshop on Networking Meets Databases (NetDB'06), 2006.

[22] V. S. W. Eide, F. Eliassen, O.-C. Granmo, and O. Lysne, "Supporting timeliness and accuracy in distributed real-time content-based video analysis," in Proceedings of the ACM International Multimedia Conference and Exhibition, pp. 2132, 2003.

[23] Y. Chi, P. Yu, H. Wang, and R. Muntz, "Loadstar: a load shedding scheme for classifying data streams," in Proceedings of the IEEE International Conference on Data Mining (ICDM '05), October 2005.

[24] V. Kumar, B. F. Cooper, and K. Schwan, "Distributed stream management using utility-driven self-adaptive middleware," in Proceedings of the International Conference on Autonomic Computing (ICAC'05), pp. 3-14, 2005.

[25] E. Horvitz and G. Rutledge, "Time-dependent utility and action under uncertainty," in Proceedings of the 7th Conference on Uncertainty in Artificial Intelligence, pp. 151-158, July 1991.

[26] M. Puterman, Markov Decision Processes: Discrete Stochastic Dynamic Programming, John Wiley \& Sons, New York, NY, USA, 1994.

[27] R. Sutton and A. Barto, Reinforcement Learning: An Introduction, MIT Press, Cambridge, Mass, USA, 1998.

[28] D. Bertsekas, Nonlinear Programming, Macmillan, New York, NY, USA, 1997.
[29] E. Crawford and M. Veloso, "Learning to select negotiation strategies in multi-agent meeting scheduling," in Proceedings of the Working Notes of the Multiagent Learning Workshop, pp. 27-33, AAAI, Pittsburgh, Pa, USA, July 2005.

[30] A. O. Hero and J. K. Kim, "Simultaneous signal detection and classification under a false alarm constraint," in Proceedings of the IEEE International Conference on Acoustics, Speech and Signal Processing (ICASSP '90), vol. 5, pp. 2759-2762, Albuquerque, NM, USA, April 1990.

[31] D. S. Turaga and T. Chen, "I/P frame selection using classification based mode decision," in Proceedings of the IEEE International Conference on Image Processing (ICIP '01), vol. 3, pp. 550-553, 2001.

[32] N. Cristianini and J. Shawe-Taylor, An Introduction to Support Vector Machines, Cambridge University Press, Cambridge, UK, 2000.

[33] D. Bertsekas and R. Gallager, Data Networks, Prentice-Hall, Englewood Cliffs, NJ, USA, 2nd edition, 1991.

[34] S. D. Viglas and J. F. Naughton, "Rate-based query optimization for streaming information sources," in Proceedings of the ACM SIGMOD International Conference on Management of Data, pp. 37-48, 2002.

[35] R. G. Gallager, Discrete Stochastic Processes, Kluwer Academic Publishers, Dordrecht, The Netherlands, 1996.

[36] P. Burke, "The output of a queuing system," Operations Research, vol. 4, p. 699, 1956.

[37] D. Gross and C. Harris, Fundamentals of Queueing Theory, Wiley-Interscience, New York, NY, USA, 1997.

[38] J. Nash, “The bargaining problem," Econometrica, vol. 18, no. 2, pp. 155-162, 1950.

[39] M. Ciraco, M. Rogalewski, and G. Weiss, "Improving classifier utility by altering the misclassification cost ratio," in Proceedings of the 1st International Workshop on Utility-Based Data Mining, 2005.

[40] J. R. Smith, "IBM multimedia analysis and retrieval system (MARVEL)," http://mp7.watson.ibm.com/marvel.

[41] L. Xie, S.-F. Chang, A. Divakaran, and H. Sun, "Structure analysis of soccer video with hidden Markov models," in Proceedings of the IEEE International Conference on Acoustics, Speech, and Signal Processing (ICASSP '02), vol. 4, pp. 40964099, Orlando, Fla, USA, May 2002.

[42] C. A. O'Cinneide, "Entrywise perturbation theory and error analysis for Markov chains," Numerische Mathematik, vol. 65, no. 1, pp. 109-120, 1993.

[43] D. Fudenberg and J. Tirole, Game Theory, MIT Press, Cambridge, Mass, USA, 1991.

[44] M. Campbell, A. Haubold, M. Liu, et al., "IBM Research TRECVID-2007 Video Retrieval System". 